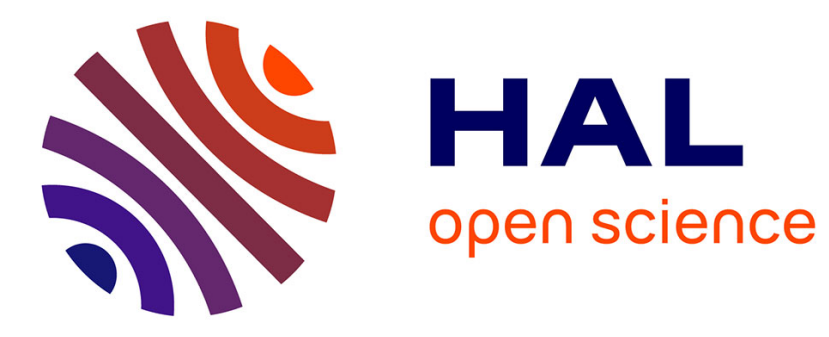

\title{
Actualité de l'enquête et des études sur l'oral
}

Claire Blanche-Benveniste, Jean-Claude Chevalier, Céline Dumont, Pierre

Encrevé, Clive Perdue, Marie-Rose Simoni-Aurembou, Gabriel Bergounioux

\section{To cite this version:}

Claire Blanche-Benveniste, Jean-Claude Chevalier, Céline Dumont, Pierre Encrevé, Clive Perdue, et al.. Actualité de l'enquête et des études sur l'oral. Langue française, 1992, 93 (1), pp.94 - 119. 10.3406/lfr.1992.5813 . halshs-01842509

\section{HAL Id: halshs-01842509 \\ https://shs.hal.science/halshs-01842509}

Submitted on 18 Jul 2018

HAL is a multi-disciplinary open access archive for the deposit and dissemination of scientific research documents, whether they are published or not. The documents may come from teaching and research institutions in France or abroad, or from public or private research centers.
L'archive ouverte pluridisciplinaire HAL, est destinée au dépôt et à la diffusion de documents scientifiques de niveau recherche, publiés ou non, émanant des établissements d'enseignement et de recherche français ou étrangers, des laboratoires publics ou privés. 


\section{Actualité de l'enquête et des études sur l'oral}

Gabriel Bergounioux, Claire Blanche-Benveniste, Jean-Claude Chevalier, Céline

Dumont, Pierre Encrevé, M. Clive Perdue, Marie-Rose Simoni-Aurembou

\section{Citer ce document / Cite this document :}

Bergounioux Gabriel, Blanche-Benveniste Claire, Chevalier Jean-Claude, Dumont Céline, Encrevé Pierre, Perdue Clive, Simoni-Aurembou Marie-Rose. Actualité de l'enquête et des études sur l'oral. In: Langue française, $n^{\circ} 93,1992$. Enquête, corpus et témoin. pp. 94-119;

doi : https://doi.org/10.3406/lfr.1992.5813

https://www.persee.fr/doc/lfr_0023-8368_1992_num_93_1_5813

Fichier pdf généré le 05/05/2018 


\section{ACTUALITÉ DE L'ENQUÊTE ET DES ÉTUDES SUR L'ORAL}

Table ronde, tenue le samedi 12 janvier 1991 à Paris, avec Jean-Claude Chevalier comme modérateur, et les participants suivants :

Claire Blanche-Benveniste, professeur à l'Université d'AixMarseille I

Jean-Claude Chevalier, professeur à l'Université Paris-VIII

Céline Dumont, maître de conférences à l'Université d'Orléans

Pierre Encrevé, professeur à l'Université Paris-VIII

Clive Perdue, maître de conférences à l'Université Paris-VIII

Marie-Rose Simoni-Aurembou, directeur de recherches au C.N.R.S.

Gabriel Bergounioux, maître de conférences à l'Université d'Orléans.

La transcription, la sélection et le découpage des interventions ont été effectués par G. Bergounioux avec l'accord de chacun des participants. La publication représente environ le quart du temps de parole total.

Après une rapide présentation des participants, Jean-Claude Chevalier ouvre la discussion.

\section{Il y a spécificité de l'oral...}

\section{J.-C. Chevalier}

Un constat pour commencer, et qui est une des raisons de notre présence : les recherches sur l'oral paraissent toujours plus ou moins marginales, ceux qui les font se sentent plus ou moins minorés. Il y aurait, visiblement, un lieu particulier pour l'enquête qu'on pourrait tenter de cerner en posant trois problèmes essentiels :

- le premier sur le rapport oral / écrit : quelle est la spécificité de l'oral et de la méthode conditionnée par le domaine? Le problème est aussi bien historique qu'idéologique.

- Deuxièmement, faire un bilan sur ce que nous avons appris de ces recherches qui ont un siècle : qu'a-t-on appris de l'oral que ne nous aurait pas appris l'écrit? 
- Et enfin, troisième point, concernant les sujets de la science : qui fait de l'oral ? Pourquoi y a-t-il des gens qui s'attachent à l'oral et d'autres pas ? Qui choisit-on comme sujet de ces analyses, comment choisit-on ses témoins?

Alors je lance le débat sur le premier point : y a-t-il une spécificité du domaine intitulé oral et, deuxièmement, y a-t-il une spécificité de la méthode d'approche de l'oral par rapport à l'écrit? Claire, puisque tu viens de publier un livre au CNRS sur la question?

\section{Blanche-Benveniste}

Il y a d'abord une spécificité évidente dans tout ce qui est intonatif, morpho-phonologique, et qui n'a pas été forcément le plus étudié. Ainsi, $\mathrm{j}$ 'en suis venue à me demander si on pouvait concevoir des morphophonologies complètement différentes avec des syntaxes qui resteraient assez proches, du fait qu'il y a spécificité de l'oral dans tout ce qui est de l'ordre de la tonologie, de la morpho-phonologie et très peu du côté de ce qu'on pourrait appeler la syntaxe (avec tous les problèmes de délimitation du fait syntaxique, bien sûr). Je ne suis pas sûre qu'il y ait une spécificité syntaxique. Pour moi, je dirais non... a priori.

Mais j'imagine un domaine très variable, avec des spécificités dont certaines ne sont peut-être pas très bien dégagées, comprenant les traits de morpho-phonologie tels que les liaisons, les formes morphologiques des verbes, les marques de féminin, de pluriel, la réalisation des morphèmes relatifs...

\section{P. Encrevé}

Il y a aussi beaucoup de spécificités du point de vue pragmatique. À l'évidence, la différence entre l'écrit et l'oral c'est que l'écrit est monologique, alors qu'à l'oral, il y a toujours présence d'un autre, plus ou moins. Ce qui se joue d'ostension, par exemple, ne s'inscrit pas de la même manière à l'écrit.

Peut-être que pour bien parler des spécificités de l'oral, il faut parler des spécificités de l'écrit. L'écrit peut se transporter dans d'autres situations, son autonomisation est très particulière. Le travail linguistique ne peut pas se passer des deux ; parce que c'est dans la comparaison qu'il met en évidence la spécificité de l'un et de l'autre.

D'ailleurs, par définition, il y a enquête sur toutes les formes que prend la langue, la forme orale, la forme écrite, avec peut-être des choses intermédiaires, par exemple l'accompagnement de l'image. J'anticipe, 
mais c'est uniquement dans ces conditions qu'on peut voir qu'il y a évidemment grammaire de l'oral.

\section{J.-C. Chevalier}

Tu dis que l'écrit est un monologue mais toutes les recherches depuis Bally ont montré que tout texte écrit était dirigé vers un sujet...

\section{P. Encrevé}

Le monologue, c'est toujours ça ! Ça s'adresse toujours à quelqu'un, même Robinson sur son île. Ce que je dis, c'est qu'il n'y a pas le rapport dialogique, il n'y a pas question-réponse. C'est ça la vraie grande différence : la possibilité d'intervention de celui qui est en face.

\section{Blanche-Benveniste}

J'aurais quelque chose à ajouter : il y a une très grande inégalité des producteurs de langage devant l'écrit, qui est moindre devant l'oral. Qui sait écrire suffisamment pour produire de l'écrit en France actuellement, et dans quelles conditions? Nous jugeons de l'oral des locuteurs et nous ne pouvons pas juger aussi librement de l'écrit du scripteur. Le problème se pose de façon très différente.

\section{Perdue}

Sur le monologue, j'aurais envie de reprendre la bonne vieille idée labovienne qu'on peut voir, dans les monologues, un certain nombre de réponses à des questions implicites. Ainsi, si on se donne les moyens d'identifier ce que pouvaient être ces questions implicites, en analysant l'étendue du discours, on peut, à ce moment-là, ramener le monologue à du dialogique.

\section{P. Encrevé}

Je n’ai pas parlé de monologue mais de l'écrit monologué. Un roman, par exemple, ne répond pas à des questions. Proust monologue avec lui-même, il peut en effet répondre à des tas d'interrogations mais ça n'a rien à voir avec ce qu'on fait en moment. Si on ne montre pas la grande spécificité de l'écrit, on perd celle de l'oral. 
Quand on enquête, on voit très bien la différence. Enquêter sur l'écrit, enquêter sur l'oral, ça n'a rien à voir. Les écrits sont des objets morts qu'on rencontre quand on veut, comme on veut, on les ouvre, on les ferme, on les laisse. On ne manipule pas comme ça l'oral.

\section{M.-R. Simoni-Aurembou}

Du point de vue de la géolinguistique, que je représente, l'étude de l'intonation est parfaitement pertinente pour rendre compte de variations géographiques, mais il faut recourir, comme le dit Fernand Carton, au laboratoire : la qualité des enregistrements de terrain, et les conditions mêmes dans lesquelles ils sont effectués font qu'ils se prêtent rarement à une analyse phonétique poussée.

Je voudrais insister sur un autre fait : dans la géolinguistique, il y a un savoir qui n'existe que dans l'oral, le savoir lexical. Il n'a jamais été écrit : c'est le savoir ordinaire des mots ordinaires, du quotidien; il n'a jamais été pris en compte par des textes écrits et on a bien du mal à identifier les unités, d'où l'importance de l'étymologie dont on ne peut pas se passer.

\section{P. Encrevé}

Oui, d'ailleurs pourquoi voit-on si mal la différence entre l'oral et l'écrit ? Parce que nous avons en général accès à l'oral sous forme d'écrit, c'est-à-dire transcrit. Et on oublie que la transcription de l'oral ne permet pas de travailler sur l'oral. Une fois qu'on a écrit de l'oral, c'est de l'écrit ! et la spécificité de l'écrit, c'est d'être de la langue déjà représentée.

Autre chose, l'écrit peut être rhabillé. Racine, rhabillé dans l'orthographe d'aujourd'hui, c'est toujours Racine et un des grands problèmes de l'orthographe, en France, c'est que les gens ont l'impression que rien n'a jamais bougé puisqu'on lit Racine dans l'orthographe d'aujourd'hui. On ne peut pas faire ça avec l'oral. L'écrit est manipulable, l'oral ne l'est pas - pas de la même manière du moins, puisqu'il perd toute sa spécificité dès qu'on le transcrit.

Le français oral n'est pas dit en alphabet phonétique!

\section{Y a-t-il une grammaire de l'oral ?}

\section{J.-C. Chevalier}

Seconde question, suivant vos interventions : « $Y$ a-t-il une grammaire de l'oral ? ». On a admis qu'il y avait des problèmes d'intonation, 
des lexiques, dans les patois, qui n'étaient pas relevés, des faits de pragmatique, mais on n'a pas démontré qu'il y avait une grammaire de l'oral. Les termes eux-mêmes sont paradoxaux : « gramma » signifie la lettre, la grammaire par définition se soutient de l'écrit. Il faudrait se justifier d'écrire une grammaire de l'oral dès lors qu'il y a contradiction dans les termes.

\section{P. Encrevé}

Tu as raison. Puisque tu dis « gramma », renvoyons à la Grammatologie de Derrida et à son concept d'archi-écriture : derrière tout oral, il y a une écriture. Les sciences cognitives vont dans ce sens, et déjà, déjà Chomsky. En français, il y a vraiment deux grammaires. Un enfant qui apprend l'orthographe écrit «héroïne et «héros » avec un «h »; pourtant, quand il parle, il dit «l'héroïne » et « le héros ». Inversement, « onze », sans «h », sera dit non pas « l'onze » mais « le Onze de France ». Ces deux codages compliquent la vie aux enfants parce qu'ils sont obligés de construire une grammaire de l'écrit et une grammaire de l'oral extrêmement différentes.

Il y a une archi-écriture, si l'on me permet de me réapproprier ce terme au sens de la phonologie cognitive : l'oral est prononciation d'une écriture interne qui est complètement différente de l'écriture externe. Et s'il y a archi-codage, archi-gramme, il y a grammaire.

Et celle-ci sera spécifique de l'oral, en morpho-phonologie et aussi en sémantique-pragmatique, comme l'épreuve « référence ostensive »le montre à l'évidence. Elle le sera aussi en ce qu'elle diffère de l'écrit littéraire.

Une deuxième chose : dans les sociétés sans écriture, il existe une formidable stylistique. Par exemple, les Fon avaient, paraît-il, une langue des nobles différente de la langue des esclaves qui leur parlaient, puisque les nobles parlaient de façon toujours rythmée, le roi ne s'adressant qu'en l'équivalent du vers, soit un codage typique du genre littéraire.

\section{J.-C. Chevalier}

Il y a des grammaires. Qu'il y ait une grammaire de l'oral, c'est une nécessité puisque autrement on ne parlerait pas. La question demeure : y a-t-il une grammaire spécifique de l'oral qu'on pourrait opposer à une grammaire de l'écrit?

Quant au fait que des sociétés sans écriture aient des parlers différents, le phénomène est bien connu mais l'exemple donné prouve-t-il 
l'existence d'une archi-écriture? Quelle serait sa représentation ? Quel rapport y aurait-il avec une grammaire de l'écrit dès lors que la grammaire des langues non écrites n'est pas explicite?

\section{Blanche-Benveniste}

Dans toutes ces sociétés, il y a des maîtres de langage. Quand on demande des renseignements sur la langue, on nous adresse à eux parce qu'ils peuvent expliquer, et d'abord attester, les formes.

\section{J.-C. Chevalier}

Il y a une sensibilité à des différences, à des codes. Mais peut-on appeler grammaire un code ? Ce serait une définition singulièrement extensive. Où est la spécificité d'une grammaire de l'oral ?

\section{P. Encrevé}

Très spécifique est l'opposition ponctuation / intonation, en plus de tout ce qu'on a dit. Et puis, toutes les fautes, au sens normatif, qui passent beaucoup plus dans l'oral que dans l'écrit. La surveillance n'étant pas la même, la variabilité par rapport au code normatif est beaucoup plus grande à l'oral.

\section{Perdue}

Essayons de poser la question autrement. Quand on parle de syntaxe, généralement, on parle de quelque chose de relativement localisé, qui ne va pas au-delà de la proposition. Mais est-ce qu'une organisation qui, à travers des énoncés, organise le discours mériterait le nom de syntaxe, du fait de sa régularité, si différente de ce qui se joue dans l'écrit?

\section{Blanche-Benveniste}

Pour être plus précise, je ne sais pas s'il y a une spécificité générale. Il y a peut-être des spécificités individuelles. Mais si on prend un ensemble de gens qui écrivent et un ensemble de gens qui parlent, la grammaire qu'on croit spécifique de l'oral, même au-delà des limites de la phrase, jusqu'au paragraphe, à l'organisation d'ensemble, va se retrouver immanquablement dans un texte publié. Quand on fait la chasse à ce qui pourrait être un exemple spécifique, même individuel, d'organisation de l'oral, syntaxique ou au-delà du syntaxique, il est difficile de ne pas trouver un 
contre-exemple immédiatement, chez tel journaliste, tel écrivain, tel scripteur maladroit, même. Il faudrait préciser la question.

\section{G. Bergounioux}

Pour prendre un exemple historique, à un moment donné, les scribes, en France, ont écrit une langue dans laquelle ils maintenaient les cas, alors que le français parlé, au moyen âge, ne les utilise plus, ou pratiquement plus. On sait que Marguerite Durand avait une intuition comparable, qui ne s'est pas avérée, selon laquelle les genres disparaîtraient en français parlé. On voyait apparaître la spécificité d'une grammaire de l'oral à travers ce type de discordance morphologique.

Est-ce qu'aujourd'hui, nous, qui faisons dans l'oral, nous avons perçu quelque chose d'aussi caractéristique?

\section{M.-R. Simoni-Aurembou}

L'oral permet d'étudier la variation et de pressentir des changements grammaticaux, dès lors qu'on dispose de témoignages. Pour le moyen âge, on peut le reconstituer, avec bien des difficultés, et plus facilement maintenant, dès lors que l'on commence à avoir des enquêtes sur cent ans.

En Suisse, à l'Étivaz, Jaberg a refait l'enquête d'Edmont. Il constate des divergences mais, au lieu de s'en tenir à ce simple constat, comme tant d'autres critiques de l'Atlas Linguistique de la France, et de conclure « il a tort et $\mathrm{j}$ 'ai raison ", ce sera pour lui l'occasion de réfléchir sur la méthodologie de l'enquête et de dégager deux catégories : la notation « impressionniste » et la notation schématisée.

Autre cas. Lanslebourg, en Savoie, a été enquêté quatre fois en soixante ans, par Edmont, puis Duraffour, sur le terrain, par Clédat par correspondance, enfin par Gaston Tuaillon pour l'Atlas du Jura et des Alpes du Nord. C'est à partir de ces quatre notations, dans leurs convergences et leurs divergences, que Tuaillon a pu conclure à l'existence d'une variation morphologique, le polymorphisme des infinitifs issus de -ARE latin.

Bien sûr, ce n'est pas une grammaire totalement spécifique, puisqu'on retrouve des terminaisons verbales, des paradigmes.

Pour revenir à la question du changement, on observe naturellement dans l'oral des faits en germe, qui vont se réaliser, mais finalement, si on prend le problème par l'autre bout, ce serait la même chose dans l'écrit et dans l'oral. C'est d'ailleurs la conclusion de Claire Blanche-Benveniste.

\section{Blanche-Benveniste}

Je voudrais répondre à la question de tout à l'heure. Je pense qu'on pourrait voir, chez beaucoup de locuteurs, une amorce de changement du 
pluriel dans les syntagmes nominaux. Lorsque les gens se mettent à dire : "Les Sud-z-Américaines », ou «des machines à laver-z-anglaises », que c'est massif, il y a peut-être une évolution. Et ça ne passera pas à l'écrit, pour des raisons techniques, orthographiques. C'est pourquoi la morphologie me paraît être un secteur différent, du fait de la variabilité plus grande de l'oral, alors que dans le domaine syntaxique, dont je ne connais pas bien les limites, le problème est assez différent.

\section{P. Encrevé}

Je crois simplement que toute grammaire est hétérogène. Ceux qui disent : «Il n'y a de grammaire que de l'écrit », veulent nous enfermer dans l'homogénéité d'une grammaire. En réalité, toute grammaire d'un locuteur est hétérogène, avec une extrême variabilité et c'est vrai que, d'une certaine façon, l'écrit est à un bout de la variabilité et l'oral plutôt à l'autre.

Simplement, si la grammaire de l'écrit et de l'oral sont de même type, avec des marges de variation plus grandes à l'oral, il ne faut pas oublier qu'on n'a accès qu'aux productions du locuteur et du scripteur. Or je pense qu'il est fondamental d'intégrer au concept de grammaire les grammaires d'auditeur et de lecteur ! Ce sont aussi des grammaires d'une extrême hétérogénéité. Quand on lit à la fois Céline et Bossuet, on est face à deux normes. Je ne pense pas qu'il y ait une spécificité complète de la grammaticalité de Céline par rapport à celle de Bossuet, mais il y a de fortes divergences. Finalement - mis à part le problème très spécifique de l'orthographe - c'est quand même une seule grammaire du français, avec une très grande variabilité et c'est ce sur quoi je conclurai : oui, les locuteurs ont une seule grammaire mais elle est très fortement hétérogène. Pour le Saussure du Cours de Linguistique Générale, il n'y a langue que de ce qu'on intériorise à partir de l'audition, et non de ce qu'on produit.

\section{J.-C. Chevalier}

Cette extrême variabilité expliquerait que les grammaires qui ne concernent que l'oral apparaissent tardivement. On pourrait se poser la question : pourquoi est-ce seulement à certaines époques qu'on voit apparaître des grammaires fondées sur l'oral ? C'est une question familière à Culioli.

\section{P. Encrevé}

On a pris grammaire au sens du "gramme », c'est-à-dire de l'écrit. Par définition, on a dit : « La grammaire, c'est de l'écrit ». Mais, au fur et 
à mesure qu'il y a eu des linguistes, ils ont entrepris d'étudier aussi le codage de l'oral. Le moment le plus intéressant, c'est la naissance de l'écriture. Quand ça commence, de la part de ceux qui en sont au principe, l'idée que l'on peut écrire, c'est la découverte de l'archi-écriture. Il y a un codage implicite, une " écriture 》 intériorisée, une « représentation » mentale de la langue qui autorise l'utilisation d'une écriture extériorisée. On passe d'une «archi-trace » à des tracés dans l'argile. Je voudrais ajouter que l'écriture n'a pas commencé avec les pictogrammes, mais avec des signes abstraits, que ce soit à Sumer ou ailleurs. Cette abstraction me paraît liée à la réalité mentale de l'archi-écriture.

\section{Quand il y a eu du vrai parlé, on ne s'y est pas intéressé}

\section{Blanche-Benveniste}

Il se peut que nous soyons dans une forme de culture qui s'occupe peu de ces échanges oraux en dehors des zones techniques. En France, on ne forme même pas les gens à une discipline de prise de parole autour d'une table. Il n'y a pas un intérêt pour les échanges de paroles aussi fort que dans d'autres cultures.

\section{J.-C. Chevalier}

Il y aurait une sacralisation de l'écrit qui serait un phénomène français?

\section{M.-R. Simoni-Aurembou}

Oui, d'ailleurs, dans tous les projets de la future Bibliothèque de France, il est intéressant de voir comment l'oral, la spécificité du document oral, n'est pas prise en considération. Au mieux, c'est perçu comme un complément de l'écrit.

\section{P. Encrevé}

Pourquoi a-t-on focalisé sur l'écrit ? Parce qu'il est moins nombreux que l'oral. L'extrême problème de l'oral, c'est qu'il est partout, il est à l'infini. C'est parce qu'il y a trop d'oral qu'on ne peut pas le conserver. Le problème, c'est la profusion. 
J'enchaîne sur un fait très important. Il n'est pas vrai que le monde scolaire soit l'univers de l'écrit. Simplement, comme on n'y pratique pas l'étude de l'oral, on ne prend pas garde qu'à l'université, à l'école, l'oral est quantitativement dominant.

Il y a cependant une éducation à la liberté de parole, en France, même si elle reste implicite. Notre société la permet.

\section{J.-C. Chevalier}

En somme, il y a un problème de rareté et de commodité de travail du fait que l'écrit apparaît comme une exception dans le monde dévorant de l'oral. C'est un lieu d'étude plus simple, du moins plus élémentaire. Il y aurait peut-être un phénomène à rapprocher par exemple de l'opposition de la peinture et de la photographie.

Un autre problème se poserait, celui de la nature. L'écrit apparaît comme un artifice, plus facile à étudier, et l'oral comme davantage lié à la nature, avec une extrême variabilité, insaisissable. Il y aurait peut-être un problème idéologique : tout ce qui est trop proche de la nature et de ses multiples variations n'est pas objet de science.

Je reviens à un point de vue historique. On a peu de travaux de gens qui ont décrit l'oral. Même s'ils disent qu'ils s'appuient sur l'oral, souvent ils ne font que de l'écrit. Antoine le remarquait justement pour Charles Bally, fondateur d'une stylistique de l'oral, qui prenait ses exemples dans Labiche.

On assiste à un phénomène de refoulement intéressant. Même si Malherbe dit qu'il faut parler comme des crocheteurs du Port-au-foin, même si Vaugelas dit : "Il faut s'inspirer de la façon dont on parle ", on ne sait pas, ou à peu près pas, comment on parlait au XVII ${ }^{e}$ siècle. On ne l'a pas noté ; il y a donc un problème de refoulement qui n'est peut-être pas seulement un problème de commodité.

\section{P. Encrevé}

L'objet n'est pas constitué puisqu'il n'est pas déjà écrit. Il n'y a qu'un demi-siècle qu'on peut conserver l'oral sans l'écrire. Le jour où le magnétophone se répand, l'oral peut être étudié comme oral. Avant, l'oral n'est étudié que comme écrit, et le problème est d'avoir des écritures proches de ce que le linguiste entend.

\section{M.-R. Simoni-Aurembou}

C'est le cas, au XVII', des partisans d'une orthographe réformée : Vaudelin, Meigret, d'Olivet... 


\section{P. Encrevé}

Et au XIX $X^{e}$, Passy, toutes les tentatives d'écriture phonétique. À ce moment-là, il n'y avait d'archivage possible de l'oral que par écrit. L'écrit peut être travaillé comme tel. En revanche, l'oral, il faut d'abord l'écrire pour le travailler.

Pendant très longtemps, les linguistes n'ont travaillé sur l'oral qu'une fois qu'ils l'avaient transcrit. J'ai passé mon temps à déconseiller à mes étudiants de commencer par transcrire. Si on transcrit de l'oral, on en fait de l'écrit. Il faut absolument au contraire réussir à garder à l'oral toute son extraordinaire spécificité, toutes ces marques qui ne s'écrivent pas, même dans les alphabets phonétiques les plus complets.

Par exemple, je pense qu'il est très important de publier chaque fois qu'on peut des spectrogrammes, tout ce qui rend quelque chose du son. Ce sont des représentations, aussi, mais pas des représentations écrites ; elles permettent de voir que c'est tout à fait autre chose que de l'écrit. Il est très très important de garder la différence.

L'oral écrit, ce n'est plus de l'oral. Il a perdu son corps, il n'a plus que ce squelette, nécessaire mais insuffisant, qu'est l'écriture. Tout ce qui est le corps, comme le corps d'un humain, ses aspects spécifiques, ne peut pas être appréhendé une fois qu'on a transcrit.

\section{J.-C. Chevalier}

Il n'empêche. L'expérience prouve que l'étude de l'oral sans transcription est extrêmement difficile, quasi-impossible même. On a énormément de peine à interpréter. Et ça repose le problème de l'élaboration d'une grammaire de l'oral.

\section{M.-R. Simoni-Aurembou}

Ce qui prouve qu'il faut absolument conserver le document oral. Bon. Mais on est confronté au désintérêt des Français vis-à-vis de l'oral. Je pense aux documents anciens qui sont à la Phonothèque Nationale, et pour le catalogue desquels j'ai travaillé : par exemple, avant 1980 , le fonds de l'Atlas Sonore de la France, commencé par Ferdinand Brunot avant 1914, n'avait pas été catalogué; les enregistrements n'étaient donc pas consultables, et avaient en effet été très peu consultés.

Un autre point intéressant : on est très déçu par une bonne part des discours des hommes politiques. On se dit : « Ah, je vais entendre la voix 
de Dreyfus ». Très bien. On entend la voix de Dreyfus, mais ce n'est pas de la parole... Les hommes politiques lisent des discours, des discours écrits et qu'ils ont prononcés. Dreyfus lit une partie de ses Mémoires... puisque c'est encore bien mieux d'avoir de l'oral quand on le lit. C'était la conception de 1912. Il n'y a que Ferdinand Brunot à avoir une conversation avec un artisan du Faubourg Saint-Antoine.

Charles Bruneau aussi, quand il a fait son enquête dans les Ardennes avec Ferdinand Brunot (c'est une partie de l'Atlas Sonore) a posé un questionnaire aussi libre que possible, dans la mesure où il avait deux minutes d'enregistrement par disque, contrainte que l'on oublie souvent. Ces disques ne sont d'ailleurs pas toujours de bonne qualité, car il ne semble pas qu'Émile Pathé ait fourni un matériel de premier choix.

\section{J.-C. Chevalier}

Ce qui confirme qu'il n'y a pas seulement un problème de commodité, mais aussi un problème de refoulement de l'oral lié à la nature, lié au corps. On retrouve le même problème que pour les patois. On ne s'intéresse pas aux patois en soi, mais on s'y intéresse en tant que ça permet d'apporter des éléments, comme on le disait en parlant de la spécificité d'une grammaire de l'oral, pour l'étude de cas par ailleurs soigneusement définis par rapport à l'écrit. On peut suivre des genèses, des évolutions, c'est une sorte d'additif intéressant.

Un problème de refoulement qu'on retrouve dans le fait que, de façon générale, les autorités ne veulent pas de ces textes oraux. C'est un problème social qui est en jeu.

\section{Blanche-Benveniste}

Je signalais le problème de l'école tout à l'heure : je ne sais pas si l'école s'est fixé un objectif de travail sur l'oral. Elle s'est fixé beaucoup d'objectifs, nécessairement, de travail sur l'écrit, d'acquisition de modes d'écritures mais se fixe-t-elle des modèles de production de l'oral ? C'est une question. Peut-être plus dans l'enseignement technique que dans l'enseignement classique, c'est possible. Mais l'école ne peut pas s'intéresser à l'oral si elle n'a pas un objectif qui sous-tend sa démarche et constitue l'oral comme objet d'étude.

\section{Le chomskysme, c'est typiquement de l'enquête orale}

\section{G. Bergounioux}

Je rejoins ce qui a été dit sur le refoulement. J'ai l'impression que si on parle de l'école, on se dédouane un peu en tant que linguistes en disant : 
« C'est bien la faute à l'école si... » On a quand même des responsabilités dont l'une pourrait être l'interprétation qu'on a eue, en France, du structuralisme, c'est-à-dire la manière dont on en a fait quelque chose qui ne requérait plus d'aller directement prendre du texte. C'est d'autant plus curieux que Martinet est a priori un contre-exemple, je pense à sa première enquête dans les camps de prisonniers, dans la ligne d'un projet de Ferdinand Brunot pendant la Première Guerre Mondiale. Quand on regarde ensuite ce que devient, en France, le structuralisme en linguistique, on a l'impression que c'est un structuralisme qui refoule et la variation, et le corps.

\section{P. Encrevé}

C'est légitime, du point de vue du structuralisme. Que fait le structuralisme? Il dit : «On va retenir, dans le son, ce qui est fonctionnel. " L'invention de la phonologie, c'est l'abandon du corps. Il est tout à fait normal que le structuralisme ait conduit à ne plus écouter le corps puisqu'il redécouvre à sa manière l'archi-écriture. Au-delà des manifestations variables à l'infini et inanalysables du corps de la langue, il y a, dit le structuralisme, un squelette qui renvoie, on le comprend mieux aujourd'hui, à l'archi-écriture mentale.

Aujourd'hui, on voit bien le chemin, à partir de la phonologie générative, la phonologie post-générative, jusqu'au travail des phonologies cognitivistes. On cherche quel est le codage, ultimement neuronal, s'il y en a un. Le structuralisme, c'est le moment où la linguistique se débarrasse du corps.

Je voudrais revenir sur le chomskysme. Le chomskysme, s'agissant de la constitution des données, c'est typiquement de l'enquête orale sur de l'oral. Les sociolinguistes nous ont conduits à ne pas comprendre vraiment ce que peut vouloir dire l'enquête. L'enquête, ce n'est pas seulement enregistrer les gens dans la rue. C'est aussi ce que fait Chomsky, c'est-à-dire poser des questions de grammaticalité. C'est une forme de l'enquête orale, d'ailleurs passionnante. Je suis de ceux qui pensent que les deux types d'enquête (enregistrement, questionnement) sont strictement indispensables.

J'ai été frappé par le fait que, quand on s'adresse à des patoisants, ils posent des jugements de grammaticalité sur le patois. Si on fait des jugements de grammaticalité sur une langue non écrite, c'est qu'il y a une grammaire de l'oral.

\section{M.-R. Simoni-Aurembou}

Pour nous, géolinguistes, l'étude des jugements de grammaticalité, du métalangage, constitue l'après-Atlas. Ce sont les recherches sur la 
conscience linguistique, qui succèdent aux enquêtes avec questionnaire strict, et requièrent un nouveau type de questionnaire.

Il n'y a pas UNE enquête. C'est bien difficile d'approcher la réalité linguistique. Il y faut une multiplicité de questionnaires.

\section{P. Encrevé}

Au fond, notre problème, c'est : est-ce que, à part la phonologie, il y a un travail scientifique sur l'oral à faire? L'évolution de Labov va dans le sens que $j$ 'indiquais tout à l'heure : ne plus se contenter de transcrire. Dans ses derniers travaux, il fait de la phonétique; il abandonne l'idée de faire des règles. Plus on va vers l'oral, mieux on comprend que sa spécificité est très différente de ce qu'ont cru les structuralistes. Je pense qu'il faut toujours tenter de tenir les deux bouts. Il faut viser l'archiécriture et il faut restituer le corps.

\section{M.-R. Simoni-Aurembou}

Dans l'Atlas Linguistique de l'Europe Romane qui est en cours, il y aura des cartes de phonologie, élaborées au niveau de l'Europe romane. En 1983, au congrès de linguistique romane de Trêves, Michel Contini a présenté des cartes phonologiques, à partir de paires phonologiques.

Donc, géographiquement, l'oral est cartographiable; et cartographiable au niveau de l'Europe romane, ce qui est quand même intéressant.

\section{G. Bergounioux}

Il demeure ce problème très particulier, qu'avait soulevé Culioli, qu'à l'oral, on rencontre de l'attesté qui n'est pas attestable. C'est une idée qui me semble à la fois vraie et en même temps extrêmement complexe à penser. Il semblerait qu'il y ait un débord sur lequel l'écrit, cette fois, n'aura pas prise. Ce débord est-il grammatical - en dehors des cas de lapsus -, ou l'attesté non attestable ne resterait-il pas comme résidu grammatical à traiter, comme grammaire spécifique de l'oral - je ne sais pas quel nom lui donner - , dont le statut en tout cas vaudrait d'être posé ?

\section{L'implication d'une règle descriptive et d'une règle morale}

\section{J.-C. Chevalier}

On a tourné autour de deux points. D'une part, les techniques : l'inscription de l'oral à côté de l'écrit est due à des techniques, telles que 
l'introduction du disque et concuremment de la phonétique, je veux dire de la phonétique acoustique puisque, au moment où on découvre le disque et les capacités d'enregistrement, on est capable d'analyser le phénomène physique qui est au principe, grâce à Helmholtz. Il y a une co-occurence.

Second point : le rôle que joue la nature, le corps, c'est-à-dire le lieu de l'idéologie. C'est en cela que l'école est intéressante. Je citerai Pierre Encrevé... Quand il était question d'introduire, ou de développer l'enseignement de l'oral à l'école, Pierre me disait : «Attention! Est-ce qu'on n'attente pas à la liberté des enfants sur un terrain qui leur est propre, celui de l'oral, et dont l'école va les déposséder ? » C'était un argument qui m'a semblé extrêmement important.

\section{P. Encrevé}

Pourquoi a-t-on si peu travaillé l'oral ? Il y a de bonnes et de mauvaises raisons. Il y a le refoulement, mais c'est aussi lié à la pudeur, comme, dans les livres d'école, il n'y a pas beaucoup de nus. Le corps de la langue, c'est la même chose. On expose quand on parle, et quand on se laisse aller à parler devant un micro, quelque chose de son intime et, jusqu'à un certain point, c'est désagréable. Il y a là un vrai problème de rapport au corps, de liberté, de pudeur, aussi. On peut dire « refoulement ", et on peut rappeler - ce que dirait Bourdieu - que seul l'écrit est légitime. Mais, la légitimité de l'écrit, c'est un peu comme le problème du vêtement : on est dans une société où on ne se promène pas nu. Voilà.

\section{J.-C. Chevalier}

Tu introduis une idée dont on n'a pas encore parlé, celle de « norme » puisque l'écrit est constamment lié à une norme. L'introduction d'une interprétation réglée du langage implique automatiquement - ou quasiautomatiquement - la présence de la norme. Marie-Rose SimoniAurembou y faisait allusion tout à l'heure : le sujet d'une enquête par oral a tendance à s'instaurer rapidement comme le maître de la norme.

\section{M.-R. Simoni-Aurembou}

Quand on enquête dans un village, il ne faut pas, surtout au début, aller voir le maître de langage qui est l'érudit local, parce que, pour lui, tous les autres informateurs sont mauvais, puisque c'est lui le dépositaire du savoir et qu'il est absolument inutile d'aller voir quelqu'un d'autre.

Il y a aussi des témoins terrorisants, d'excellents témoins, vers lesquels la communauté nous a dirigés, mais qui n'acceptent pas de rivaux, et peuvent même refuser leur porte aux voisins. 


\section{J.-C. Chevalier}

On en vient à se demander si l'analyse du langage ne passe pas obligatoirement par l'implication d'une règle descriptive et d'une règle morale, contrairement à ce qu'ont dit les structuralistes, par exemple.

\section{Blanche-Benveniste}

Des enfants nous ont dit : « Nous, on parle avec des fautes d'orthographe ", et ils ont refusé d'être enregistrés, à cause de ça. Ils n'ont pas tort de le dire; du moment qu'il n'y a pas l'équivalent de l'orthographe, pour eux, dans l'oral, il n'y a pas l'équivalent de norme. Dans le prolongement de ce qui avait été dit tout à l'heure, est-ce que la dérive du structuralisme n'a pas été liée au fait que l'objectif était souvent de donner des écritures aux langues. On retrouve les problèmes de gens comme Nida, de la Bible Society...

\section{Ça a été le rôle de l'oral que de faire éclater la linguistique}

\section{J.-C. Chevalier}

On en revient au problème des transcriptions qui ne sont pas des transcriptions socialisées, transcriptions de l'oral du type sonagrammes... Quel est le statut de ces transcriptions pour l'organisation de l'analyse ? Est-ce qu'il y a une différence entre enregistrer l'oral avec des lettres, ou des graphies courantes, ou au spectro, avec des analyseurs acoustiques qui permettent une certaine reproduction de la composante sonore du langage ? Est-ce qu'il n'y a pas là un nouveau code, une manière de transcription qui nous permet d'échapper à l'oral lié au corps?

\section{Perdue}

Pourtant, obligatoirement, on en reviendra à y chercher du discret.

\section{Blanche-Benveniste}

Peu de gens ont accès, directement, à ces représentations. J'avoue que je suis perturbée quand s'affichent à l'écran ces sortes de partitions où il y a tant de choses à prendre en compte. Et ces partitions, qui sait les lire couramment? 


\section{J.-C. Chevalier}

D'autant que nous sommes confrontés à des phénomènes déroutants, par exemple, les occlusives représentées par des vides, alors qu'on cherche, pour tout son, une trace tangible...

\section{P. Encrevé}

Tout dépend de l'objet, de ce qu'on cherche. Si on entreprend une recherche sur l'analyse du relatif, évidemment qu'il faut transcrire ! Même pour la liaison sans enchaînement, après un premier travail phonétique, j'ai transcrit. Il faut quand même que les choses deviennent manipulables. Simplement, le danger, c'est de faire croire que la représentation de l'oral, c'est l'oral.

\section{M.-R. Simoni-Aurembou}

L'oral, c'est le document! La transcription ne remplace jamais l'enregistrement.

\section{P. Encrevé}

D'autant qu'une fois transcrits, beaucoup de gens ne se reportent plus jamais à leurs enregistrements, ni à aucun de leurs documents d'enquête. Car il y a des tas de choses qu'on ne peut jamais enregistrer, qu'on ne peut écrire qu'en notes volantes, parce que les circonstances l'imposent... Mais tout document - y compris ceux-là, qui trahissent terriblement, qui ramènent le corps à une minuscule trace schématique est utile. Au fond, il ne faut rien rejeter, alors même qu'on est confronté à un deuxième problème: la profusion des informations. Tout est intéressant dans la langue.

\section{J.-C. Chevalier}

On arrive à un point d'explosion ! Ton intervention est caractéristique de l'état actuel de la linguistique : peut-être est-ce que ça a été le rôle de l'oral que de faire éclater la linguistique, après toutes sortes d'hypothèses formalistes qui, au contraire, avaient tendance à enlever la chair, comme le disait Pierre Encrevé, le corps? Est-ce que ça n'est pas, au moins, un élément parmi d'autres ? Si on pense aux projets concernant le redécoupage de la linguistique au CNRS par exemple...

\section{Perdue}

Peut-être que la psycholinguistique de l'acquisition, dont le développement est très lié à l'oral, apporterait ici un éclairage neuf. Pour parler 
du projet européen sur l'acquisition, quand on a essayé de déterminer ce qu'il y avait de spécifiquement acquisitionnel dans les premiers moments de fonctionnement de systèmes, il nous a manqué une base de comparaison qui était les mêmes activités langagières, enregistrées et analysées pour un locuteur natif du français, par exemple. Il a fallu faire un double travail, à ce moment-là : analyser les tentatives pour construire des discours d'une part par des apprenants du français et d'autre part, par des locuteurs natifs du milieu social où évoluaient ces apprenants.

\section{Blanche-Benveniste}

À part la psycholinguistique de l'acquisition, il y a aussi les travaux récents sur les pathologies du langage, qui sont en plein renouvellement et qui risquent de nous apprendre beaucoup. Là aussi, il faut un point de comparaison entre ce qui est réputé pathologique et ce qui est réputé ne pas l'être.

\section{Il y a un problème de vocation}

\section{J.-C. Chevalier}

Je propose que nous passions au troisième point, qui est assez sensiblement différent : pourquoi ceux qui font de l'oral, ont-ils fait de l'oral ? Qu'est-ce qu'attendaient les gens qui se sont occupés de l'oral ? Pourquoi ça et non pas, tout simplement, de l'écrit. Qui les meut ? Qui les point?

\section{M.-R. Simoni-Aurembou}

Il y a un problème de vocation. Être dialectologue, en France, hier, comme je le fus, et aujourd'hui, comme je le suis, il faut en avoir la vocation puisque c'est une discipline qu'on n'enseigne pratiquement pas à l'Université, on ne prend pas en compte la dimension de la variation dans l'espace du langage à travers les parlers locaux.

\section{J.-C. Chevalier}

Qu'est-ce que tu défendais? Qu'est-ce que tu appelles vocation?

\section{M.-R. Simoni-Aurembou}

Bon, je vais commencer par le commencement. Après avoir suivi un cursus d'espagnol très intéressant, j'ai suivi, comme mes camarades de 
Saint-Cloud, le certificat de grammaire et de philologie. Nous avions Robert-Léon Wagner et Gérald Antoine comme professeurs, et leurs assistants étaient Claude Régnier et Jean-Claude Chevalier, syntacticien qui ne m'a pas du tout intéressée à l'oral. Régnier faisait quelques cours - très rares! - sur la dialectologie au moyen âge. Les choses cheminèrent et puis, un beau jour, alors que j'avais tous mes diplômes d'espagnol, la vocation me dit : «Pourquoi ne pas chercher à voir ce qui se passe chez soi, dans son pays, dans les dialectes, dans le parler local auquel j'étais familiarisée puisque je suis née dans le Bourbonnais, au nord du Croissant ? »

Comment ça vient ? C'est un camarade qui vous donne une idée, un souvenir des dialectes au moyen âge... Mais que la dialectologie en tant que discipline existât, je n'en avais aucune idée. Et puis voilà !

Ensuite on s'aperçoit que c'est un domaine où on a sa liberté. Il n'est pas enseigné, c'est vrai... Mais la découverte du CNRS a été une vraie découverte. On me confiait un domaine, un territoire, le territoire de l'enquête. Pour un vrai dialectologue, le moment où il se voit investi d'un espace est absolument décisif. Parce que, ne l'oublions pas, la théorie de la dialectologie, c'est la carte, c'est elle qui fonde la recherche... avec le questionnaire! Mais on part de la carte!

C'est l'échiquier. Et après, on joue la partie. Et ensuite, j'ai joué ma partie. Mais c'est une vocation à... à être heureux.

\section{J.-C. Chevalier}

Et toi, Pierre?

\section{P. Encrevé}

En 1964, je suis allé voir Martinet : je voulais faire une thèse sur la poétique avec lui. 11 m'a demandé où j'étais né, ce que j'avais fait... Il m'a dit : «Quoi ? Vous êtes protestant? Né en Vendée? Je me suis toujours demandé si les protestants et les catholiques parlaient de la même manière. Vous allez m'étudier la différence entre les protestants et les catholiques dans votre village."

Je n'ai plus fait de dialectologie après, et je n'ai pas publié ma thèse sur Foussais. Je l'avais proposée à Pierre Bourdieu qui souhaitait que je développe un peu les aspects proprement sociologiques; c'était le 3 mai 68. En sortant de chez lui, j'étais sur le Boulevard Saint-Michel... En octobre, je n'avais plus la tête à ça. Et puis, de Martinet, je suis passé à Chomsky, de la Sorbonne à Vincennes. 
Je me suis peut-être laissé intimider. Je me suis passsionné pour le chomskysme et je suis devenu chomskyen ; ça ne m'intéressait plus ce que j'avais fait. Ça m'a réintéressé longtemps après, grâce à Labov. Mais j'ai eu un détour. Je me suis mis d'abord à la phonologie générative, que j'ai trouvée fascinante... que je continue à trouver fascinante.

Mais si j'ai eu mon propre mouvement d'abandon à l'égard de mon premier travail, je considère que ma thèse d'état sur la liaison, si elle ne relève plus de la dialectologie au sens étroit, est quand même une forme de dialectologie, celle des hommes politiques. Par là, j'ai renoué avec l'enquête.

J'y retrouvais un de mes plus anciens problèmes : comment faire à la fois du terrain et de la science? En France, il y a une séparation entre empirie et théorie. J'avais essayé de les renouer autour du structuralisme, puis j'avais cessé d'adhérer à cette théorie. C'est pour ça que je n'ai pas publié mon étude sur Foussais. J'ai peut-être eu tort.

\section{J.-C. Chevalier}

Et toi, Claire, ton entrée en oral ?

\section{Blanche-Benveniste}

Je pense que je me suis d'abord intéressée aux problèmes de l'orthographe, avec Chervel. Afin de voir l'autre pôle, je me suis at tachée à ce qui pouvait s'imaginer sans représentation écrite. Et puis j'ai été influencée par la familiarité, à ce moment-là, avec des linguistes africanistes et la difficulté que $\mathrm{j}$ 'avais à comprendre comment on arrivait à s'auto-présenter une langue dont on n'avait pas d'écriture, pas de dictionnaire.

J'ai trouvé tout à fait magique leur travail. Je m'en sentais complètement incapable. Mais du coup, ça m'a ramenée à ce versant, pour le français, par curiosité.

Il y avait là une autre forme de linguistique, que la syntacticienne du français ne connaissait pas du tout, qui était vraiment une linguistique de terrain. Je me demandais si ce qui se faisait correspondait à ce qu'on m'en racontait. J'avais l'impression qu'entre l'opération de magie que je voyais dans les descriptions finales, la réduction de toutes les difficultés par exemple, et ce qu'on me racontait, les choses ne correspondaient pas exactement.

\section{P. Encrevé}

Avant de travailler avec Martinet, j'avais été, à la Sorbonne, l'élève de Claire Blanche-Benveniste et $j$ 'avais été très impressionné par le fait 
que, dans les cours de grammaire et philologie, elle disait : " On ne peut pas faire de grammaire normative ", alors qu'au même moment sortait la grammaire de Wagner et Pinchon.

Je venais apprendre ce qu'était la norme grammaticale et, à tous moments, Claire disait : « Elle n'est pas fixée, elle n'est pas claire. On peut dire ça. On dit aussi ça. »

La première personne qui m'a laissé penser que la grammaire pouvait être tout autre chose que ce que j'imaginais, c'est Claire.

\section{J.-C. Chevalier}

Clive?

\section{Perdue}

Je ne suis pas sûr, après la discussion de cet après-midi, que je travaille sur l'oral. Je fais de l'analyse d'enregistrements.

En arrivant à Vincennes, j'étais étranger. Pour moi, c'est un peu l'inverse de votre démarche. Je voulais travailler sur d'autres étrangers, et il se trouve que la plupart des travailleurs immigrés sont analphabètes. C'est une raison essentielle à ma motivation pour un travail sur des enregistrements.

\section{J.-C. Chevalier}

Céline Dumont, comme tu as le dévouement de tout noter, on ne t'a pas entendue. Peut-être pourrais-tu nous dire les raisons qui t'ont menée à faire de l'oral ?

\section{Dumont}

Je me sens très concernée par tout ce qui s'est dit, que je trouve passionnant. En fait, j'ai une histoire très personnelle avec l'oral, avec la langue d'une manière générale, une histoire marquée de non-légitimité, obscurément. Je suis née dans un milieu multilingue, obligé de parler français, alors que les langues maternelles étaient l'italien, ou l'arabe, et le maltais, et le grec, dans les oreilles, dans le cœur, dans le corps. En faisant des études de lettres, je me suis un peu coupée de toutes ces racines, puis, quand j'ai essayé de solder des comptes, quelque part, avec la légitimité, l'écrit m'est apparu beaucoup trop légitime : je lui en veux et il me fascine. À l'oral, je me sens plus libre, moins sûre aussi, parce que, du côté de l'écrit, il y a les règles. Mais il reste à y surmonter tant de problèmes, du côté de l'écrit à publier, aussi... 


\section{J.-C. Chevalier}

Est-ce qu'il y aurait un lien, qui semble assez mystérieux, entre l'oral et la non publication? Est-ce que c'est lié, seulement, à la difficulté plus grande d'organiser le domaine? Dans des domaines bien balisés, bien circonscrits, il est beaucoup plus facile de mouturer des lois. Comment affronter le problème de la non écriture ?

\section{P. Encrevé}

Quand on aime vraiment l'oral, on n'a aucune raison de publier. Sauf les dialectologues qui, étant les seuls à travailler sur les dialectes, ont leur légitimité ; quand on travaille sur le français ordinaire, on se retrouve en concurrence désavantageuse avec ceux qui ont la langue légitime pour objet.

Ce qui conduit un certain nombre de gens à choisir ces objets qui sont plutôt marginalisés, c'est sans doute un rapport de soi-même à la société, qui se reproduit dans le fait qu'on a du mal à publier. S'intéresser aux objets marginaux, c'est acquiescer à une relative marginalisation de soi-même.

Pour moi, c'était plus évident de publier de l'oral d'hommes politiques, qui sont des gens qui s'exposent d'eux-mêmes, que de publier de l'oral des paysans de Foussais. J'aurais eu, avec eux, une impression d'effraction : on entre dans leur vie. Pourquoi mettre ces gens-là sur la place publique? Ils ne me l'ont pas demandé !

\section{Blanche-Benveniste}

Il peut y avoir l'inverse aussi. J'ai su par exemple que, parmi les petits extraits que nous avons publiés dans le Le français parlé : Études grammaticales, il y a cette vieille dame de Toulon qui racontait - joliment - l'histoire de sa maison. Elle m'a fait savoir qu'elle avait été très contente de voir ces quelques pages et qu'elle voulait bien reparler...

\section{On a très peu parlé de nos témoins}

\section{M.-R. Simoni-Aurembou}

La loi du 17 juillet 1970 est très stricte. Elle rappelle le droit qu'a le témoin sur sa voix et sur son image. Je vous la lis : 
« Sera puni quiconque aura volontairement porté atteinte à l'intimité de la vie privée d'autrui :

$1^{\circ}$ en écoutant, enregistrant ou transmettant au moyen d'un appareil quelconque des paroles prononcées dans un lieu privé par une personne sans le consentement de celle-ci ;

$2^{\circ}$ en fixant ou transmettant au moyen d'un appareil quelconque l'image d'une personne se trouvant dans un lieu privé sans le consentement de celle-ci.

Sera puni quiconque aura sciemment conservé, porté ou volontairement laissé porter à la connaissance du public ou d'un tiers, ou utilisé, publiquement ou non, tout enregistrement ou document obtenu à l'aide d'un des faits prévus à cet article. »

\section{G. Bergounioux}

Finalement, on a très peu parlé de nos témoins. C'est eux qui seront restés un peu dans l'ombre. Peut-être avons-nous le même problème que les psychologues. Quand on regarde des expérimentations en psychologie, ce sont des étudiants en psycho qui ont parsé les tests... Peut-on encore parler d'échantillon représentatif ? Et de quoi ?

\section{J.-C. Chevalier}

Il y aurait un problème de commodité, mais il y a aussi des techniques pour choisir : je pense aux critères des dialectologues...

\section{M.-R. Simoni-Aurembou}

Mais on ne parle quand même presque jamais des témoins.

\section{Perdue}

On est bien obligé d'en parler. Enfin, ça dépend du contexte. On ne peut pas comprendre les problèmes de l'acquisition linguistique sans parler assez longuement du témoin. Il y a là-dessus un article de P. Dalmas, dans les Cahiers du GRAL de l'Université de Provence, sur la recherche des informateurs. C'est passionnant.

\section{M.-R. Simoni-Aurembou}

L'Association Française des Détenteurs de Documents Sonores (AFAS) vient de publier l'Oral en collection, qui recense toutes les 
collections de documents sonores accessibles. Les enregistrements qui ont servi aux chercheurs pour leurs travaux n'y figurent pas souvent. Et c'est une question difficile de savoir ce que deviennent les enregistrements des chercheurs...

\section{P. Encrevé}

Je n'ai jamais donné ceux de Foussais et je ne les donnerai jamais : c'est un pacte avec les gens que $j$ 'ai vus. La première personne que j'ai rencontrée m'a dit, en voyant mon magnétophone : " Ah oui, tu veux nous enregistrer pour faire rire dans les salons parisiens. "Et elle a continué : «Tu peux enregistrer à condition que tu ne le fasses écouter à personne. " Et j'ai accepté. Après, il m'est arrivé de faire des enregistrements sans le dire, mais uniquement avec des gens de ma propre famille. Je savais que j'effacerais ce qui ne devait pas être conservé, et je leur ai dit après, naturellement. Mais je considère que c'est strictement privé.

\section{M.-R. Simoni-Aurembou}

Mais attention! Il y a des témoins qui ne veulent pas que l'on dépose l'enregistrement que l'on a fait d'eux : à ce moment-là, on ne les dépose pas. Ensuite, il y a deux autres cas :

- il y a ceux qui disent : « Mais oui, bien sûr, au contraire, je suis heureux d'avoir participé à la science. » J'ai publié des extraits de lettres, des gens qui m'écrivaient : « Je suis très heureuse que la cassette de ma voix soit déposée à la Phonothèque Nationale,... »;

- et puis, il y a ceux qui disent : « Non, pas tout de suite. »C'est le cas de tous les enregistrements sur la guerre réalisés dans le Vercors ou en Normandie, par exemple.

Maintenant, si aucun des enregistrements de Foussais n'est communicable, vous ne les déposez pas, naturellement. Mais c'est une exception parce que la masse de ce qu'on enregistre est parfaitement communicable. Cela fait même plaisir à des gens qui, comme le disait un canut lyonnais, sont « heureux de savoir que (leur) voix est en lieu sûr ». Et puis, il y a eu une évolution dans les enquêtes.

\section{P. Encrevé}

Mais oui! Non seulement, aujourd'hui, tout le monde a un magnétophone, s'enregistre, mais de plus il y a eu une évolution radicale de la structure paysanne. En 1965, quand j'ai enquêté, il y avait encore 30 ou 
$40 \%$ de paysans dans le village. Maintenant, il en reste $5 \%$. On ne voit plus le même type de personnes. On peut enquêter dans la paysannerie, mais est-ce encore une communauté linguistique?

Jean-Claude Chevalier remercie les participants et clôt la séance.

\section{BIBLIOGRAPHIE}

(Les titres qui figurent ci-dessous n'ont d'autre fin que de situer les articles et les livres mentionnés, explicitement ou non, dans la table ronde.)

Bally Charles, 1932-1933 : Traité de stylistique, Paris, Klincksieck (2 volumes).

Blanche-Benveniste Claire et Chervel André, 1969:L'orthographe, Paris, Maspero.

Blanche-Benveniste Claire et Jeanjean Colette, 1987: Le français parlé, transcription et édition, Paris, Didier Erudition.

Blanche-Benveniste Claire, avec Bilger Mireille, Rouget Christine et Van DEN EYNde Karel, 1990 : Le français parlé : études grammaticales, Paris, Editions du CNRS, Collection «Sciences du langage ».

Bourdieu Pierre, 1982 : Ce que parler veut dire, Paris, Fayard.

Calas Marie-France et Brun-Trigaud Guylaine, 1990: L'oral en collections, Paris, AFAS.

Culioli Antoine, 1983 : «Pourquoi le français parlé est-il si peu étudié ?», Recherches sur le français parlé, 5, Aix-en-Provence, Publications de l'Université.

Delmas Paul, 1983 : «Implication et enquête de terrain » in : VeroniQUe Daniel (1983).

Derrida Jacques, 1967 : De la grammatologie, Paris, Minuit.

DURAND Marguerite, 1936 : Le genre grammatical en français parlé à Paris et dans la région parisienne, Paris, d'Artrey.

EnCReve Pierre, 1967 : Problèmes de bilinguisme dialectal. La situation linguistique à Foussais (Vendée), thèse de III' cycle, Paris, Faculté des Lettres (non publiée).

EnCRevé Pierre, 1985 : «Sa voix harmonieuse et voilée ", in Hors-Cadre, 3, Saint-Denis, Presses Universitaires de Vincennes.

ENCREvE Pierre, 1976 : «Labov, linguistique, sociolinguistique », in Labov.

EnCreve Pierre, 1988 : La liaison avec et sans enchaînement, Phonologie tridimensionnelle et usages du français, Paris, Seuil.

Labov William, 1976 : Sociolinguistique, Paris, Minuit.

MARTINET André, 1945 : La prononciation du français contemporain, Genève, Droz.

NIDA Eugène, 1964: Orthographic Studies, Articles on new writing systems, Londres, United Bible Societies. 
Noyau Colette et Deulofeu José (dir.): «L'acquisition du français par des adultes immigrés (aspects sociolinguistiques), Langue Française, 71.

Perdue Clive (dir.), $1984:$ « L'acquisition du français par des adultes immigrés : aspects psycholinguistiques », Langages, 84.

Pernot Hubert, 1928 : «L'Institut de Phonétique de l'Université de Paris », Revue de Phonétique, V, 1.

Simoni-Aurembou Marie-Rose, 1974-1978 : Atlas linguistique et ethnographique de l'Ile-de-France, Orléanais, Perche, Touraine, Paris, CNRS (vol. 1 et 2).

Simoni-Aurembou Marie-Rose, 1987 : « Les entretiens enregistrés à caractère dialectologique et ethnotextuel », in Les nouvelles archives. Formation et collecte, Acte du XXVIII Congrès national des Archivistes français, Paris.

Simoni-Aurembou Marie-Rose, 1991 : « Les Atlas linguistiques. Des Linguarum totuis orbis vocabularia comparativa (1787), à l'Atlas Linguarum Europeae $(1983 \rightarrow)$ in Mémoires de la Société de Linguistique de Paris, nouvelle série, tome I.

Tuaillon Gaston, 1958 : « Exigences théoriques et possibilités réelles de l'enquête dialectologique 》 in Revue de Linguistique Romane, 22.

VERONIQUE D. (dir.), 1983 : Acquisition du français par des travailleurs marocains, Aix-en-Provence, Publications de l'Université.

Wagner Robert-Léon et Pinchon Jacqueline, 1962 : Grammaire du français classique et moderne, Hachette. 